\title{
UV consistency conditions for Cachazo-He-Yuan integrands
}

\author{
Laurentiu Rodina $\odot$ \\ Institut de Physique Theorique, Universite Paris Saclay, CEA, CNRS, F-91191 Gif-sur-Yvette, France
}

(Received 1 July 2020; accepted 2 August 2020; published 10 August 2020)

\begin{abstract}
We extend on-shell bootstrap methods from spacetime amplitudes to the world sheet objects of the Cachazo-He-Yuan formalism. We observe that the integrands corresponding to tree-level nonlinear sigma model, Yang-Mills and $(D F)^{2}$ theory are determined by demanding enhanced UV scaling under BrittoCachazo-Feng-Witten shifts. Back in spacetime, we also find that $(D F)^{2}$ theory is fixed by gauge invariance/UV scaling and simple locality assumptions.
\end{abstract}

DOI: 10.1103/PhysRevD.102.045012

\section{INTRODUCTION}

The S-matrix bootstrap program aims to construct scattering amplitudes directly from on-shell physical principles, foregoing the complicated formalisms of off-shell Lagrangians or Feynman diagrams. In this approach, the Britto-Cachazo-Feng-Witten (BCFW) recursion [1] and generalized unitarity [2] proved immensely powerful tools, enabling the calculation of otherwise intractable tree and loop level amplitudes, ultimately culminating in the purely geometric description of amplitudes in terms of geometries [3,4], while other advances uncovered a remarkable inter-connectivity between amplitudes: the double copy [5-8], transmutation operators [9], and most recently entanglement [10].

At the same time, it has been discovered that amplitudes can be fully determined with even less information than previously thought: gauge invariance or the Adler zero [11-15], Bern-Carrasco-Johansson (BCJ) relations [16], IR behavior [17], or UV scaling [16,18], together with simple locality assumptions, is each sufficient to fully constrain a wide range of tree level amplitudes, including their unitarity (factorization).

Of such constraints, UV scaling has surprisingly proven to be the most versatile and powerful: almost all theories have some hidden enhanced scaling under either single or two particle shifts, and for some of them this seems to be a defining property, as summarized in Table I.

The original motivation for studying this UV scaling was as a precondition for the BCFW recursion [1]. In this construction, the momenta of some particles are deformed by a BCFW shift $p \rightarrow p+z q$. If the amplitude has a

Published by the American Physical Society under the terms of the Creative Commons Attribution 4.0 International license. Further distribution of this work must maintain attribution to the author(s) and the published article's title, journal citation, and DOI. Funded by SCOAP ${ }^{3}$. scaling of $\mathcal{O}\left(z^{-1}\right)$ or better as $z \rightarrow \infty$, this implies the amplitude has only finite poles in $z$, and so may be rebuilt recursively from its residues, which due to unitarity (factorization) are products of lower point amplitudes. Gravity exhibits and even better scaling of $\mathcal{O}\left(z^{-2}\right)$ [19-22], which leads to "bonus relations" [23]. On the other hand, it has recently become apparent that a recursion may be setup even with poles at infinity, as long as the asymptotic behavior is sufficiently tame and can be probed by other properties [24-30], while other UV and unitarity considerations may fix gravity loop integrands [31]. However, Table I demonstrates that the UV scaling may be considered more fundamental than the recursion itself, as no other input beyond locality is needed. In terms of usual Feynman diagrams, this property is highly nontrivial to observe and requires numerous cancellations; therefore, this suggests a different formalism may exist, in which UV scaling is manifest, at the expense of the usual spacetime description following from Lagrangians.

In this paper, we find that a step in this direction is given by the Cachazo-He-Yuan formalism [32-39], which can be viewed as a transform from spacetime to a world sheet, where local singularities corresponding to propagators are replaced by world sheet singularities $\left(\sigma_{i}-\sigma_{j}\right)^{-1}$, with the precise map encoded in the scattering equations (SEs) (6). In terms of this map, amplitudes may be expressed as

$$
A=\int d \sigma \delta(\mathrm{SE}) I_{L} I_{R}
$$

where the integrands $I$ depend on the theory. Manifest locality and unitarity inherent to Feynman diagrams are lost, but gauge invariance and the double copy are more transparent in this formalism. This construction can be traced to an ambitwistor string theory [40,41], but it is not clear what (if any) principles determine the world sheet objects $I$ directly. 
TABLE I. Theories fixed by locality and specific scaling under BCFW shifts.

\begin{tabular}{lcc}
\hline \hline & Adjacent shift & Nonadjacent shift \\
\hline Yang-Mills & $z^{-1}$ & $z^{-2}$ \\
Gravity & & $z^{-2}$ \\
Born-Infeld & $z$ & $z^{0}$ \\
NLSM & & $z^{0}$ \\
Special Galileon & & $z^{2}$ \\
\hline \hline
\end{tabular}

We propose that simple scaling under BCFW shifts is sufficient to fully determine the integrands relevant for nonlinear sigma model (NLSM), YM, as well as $D F^{2}$ theory [42], even without assuming the scattering equations. The assumptions needed are that the NLSM and YM integrands are permutation invariant in all but two labels, $a$ and $b$, corresponding to the reduced rows and columns, while the integrand for $D F^{2}$ theory is fully permutation invariant, but with no dot products of the type $e_{i} \cdot e_{j}$. The only singularities come from factors $\sigma_{i j}=\sigma_{i}-\sigma_{j}$, a priori unrelated to the numerators. We also require that shifting particles $i$ and $j$ also shift the corresponding $\sigma$ 's,

$$
\begin{aligned}
\sigma_{i} & \rightarrow \sigma_{i}+z \sigma_{i}, \\
\sigma_{j} & \rightarrow \sigma_{j}+z \sigma_{j} .
\end{aligned}
$$

Under such shifts not involving reduced labels $a$ or $b$, we find that all three integrands scale as

$$
\mathcal{O}\left(z^{-2}\right)
$$

while those that do involve $a$ or $b$ may be slightly worse, as summarized in Table II.

Next, we can also consider what happens when we assume "world sheet locality": demanding that any dot product $x_{i} \cdot y_{j}$ is rescaled only by the appropriate world sheet factor $\left(\sigma_{i j}\right)^{-1}$. This provides an Ansatz that is very close to manifesting the correct scaling: for vector theories, no term scales worse than $\mathcal{O}\left(z^{-1}\right)$, so only a minor improvement is required to obtain the correct scaling of $\mathcal{O}\left(z^{-2}\right)$. Moreover, the correct scaling in most (but not all) shifts holds term by term in the expansion of the YangMills reduced Pfaffian, while the cycle expansion of the

TABLE II. Scalings under different shifts, assuming $i \neq j \neq a \neq b$, with the $a$ and $b$ corresponding to the reduced rows and columns.

\begin{tabular}{lccc}
\hline \hline & {$[i, j\rangle,[j, i\rangle$} & {$[i, a\rangle,[i, b\rangle,[a, i\rangle,[b, i\rangle$} & {$[a, b\rangle,[b, a\rangle$} \\
\hline$\left(\operatorname{Pf}^{a b}\right)^{2}$ & $z^{-2}$ & $z^{0}$ & $z^{0}$ \\
$\operatorname{Pf} \Psi^{a b}$ & $z^{-2}$ & $z^{-2}$ & $z^{-1}$ \\
$I_{D F^{2}}$ & $z^{-2}$ & & \\
\hline \hline
\end{tabular}

nonreduced Pfaffian manifests correct scaling term by term. This suggests that the world sheet is in fact a more natural home for the BCFW shift, getting us closer to a formalism which trades locality and/or unitarity for enhanced UV behavior.

The paper is organized as follows. In Sec. II, we first briefly review the Cachazo-He-Yuan (CHY) construction (a full description can be found in Refs. [32-36]). In Sec. III-V, we then present evidence that the integrands relevant for NLSM, Yang-Mills (YM), and $D F^{2}$ theory are fully determined by UV scaling and various assumptions. Motivated by this result, we also extend such observations to the usual spacetime $D F^{2}$ amplitudes, which we find to be fixed by gauge invariance and similar UV conditions. We conclude with possible future directions in Sec. VI.

\section{CHY REVIEW}

The CHY formula expresses various scattering amplitudes as

$$
A_{n}=\int d \Omega \delta(\mathrm{SE}) I_{L} I_{R}
$$

where the scattering equations are given by

$$
\mathrm{SE}_{i}=\sum_{j \neq i}^{n} \frac{p_{i} \cdot p_{j}}{\sigma_{i}-\sigma_{j}}, \quad i=\overline{1, n},
$$

and the half-integrands $I$ are functions of kinematics, polarization vectors (for vector theories), and world sheet coordinates $\sigma$. Their specific expression dictates the particular theory to be obtained, in a form manifesting the BCJ double copy. There are four ingredients relevant to our discussion, necessary to build NLSM, YM, and $D F^{2}$ theory. For all three theories, one of the integrands is the Parke-Taylor factor which encodes the ordering of the resulting amplitudes,

$$
I_{\mathrm{PT}}(1,2, \ldots, n)=\frac{1}{\left(\sigma_{1}-\sigma_{2}\right)\left(\sigma_{2}-\sigma_{3}\right) \ldots\left(\sigma_{n}-\sigma_{1}\right)} .
$$

The NLSM integrand is given by

$$
I_{\mathrm{NLSM}}=\left(\operatorname{Pf} A^{a b}\right)^{2}=\frac{(-1)^{a+b}}{\sigma_{a}-\sigma_{b}} \operatorname{det}\left(A^{a b}\right),
$$

where the reduced matrix $A^{a b}$ is obtained by removing rows and columns $a$ and $b$ from the $n \times n$ matrix $A$,

$$
A_{i j}=\frac{p_{i} \cdot p_{j}}{\sigma_{i j}}
$$

In this paper, we will focus on the simpler object $\operatorname{Pf} A^{a b}$. 
Next, the YM integrand is given by

$$
I_{\mathrm{YM}}=\frac{(-1)^{a+b}}{\sigma_{a}-\sigma_{b}} \operatorname{Pf} \Psi^{a b},
$$

where the reduced matrix $\Psi^{a b}$ is obtained by removing rows and columns $a$ and $b$ from the $2 n \times 2 n$ matrix $\boldsymbol{\Psi}$,

$$
\boldsymbol{\Psi}=\left(\begin{array}{cc}
A & -C^{T} \\
C & B
\end{array}\right)
$$

with

$$
A_{i j}=\frac{p_{i} \cdot p_{j}}{\sigma_{i j}}, \quad B_{i j}=\frac{e_{i} \cdot e_{j}}{\sigma_{i j}}, \quad C_{i j}=\frac{e_{i} \cdot p_{j}}{\sigma_{i j}} .
$$

Despite this reduction, both integrands are permutation invariant on the support of the scattering equations. We will keep track of the labels $a$ and $b$ and associate them with two particles which we single out as having a distinct scaling behavior under shifts. To avoid clutter, we will sometimes drop the extra label $a b$ from objects under consideration.

The $D F^{2}$ integrand is given by

$$
I_{D F^{2}}=\prod_{i=1}^{n} \sum_{j \neq i}^{n} \frac{e_{i} \cdot p_{j}}{\sigma_{i j}}
$$

and is directly permutation invariant. Note that the only dot products appearing are of the form $(e \cdot p)$, a fact we use as an assumption.

Other theories such as gravity, Born-Infeld, or the special Galileon may be obtained by mixing these ingredients,

$$
\begin{gathered}
\mathrm{GR} \sim I_{\mathrm{YM}} I_{\mathrm{YM}}, \\
\mathrm{BI} \sim I_{\mathrm{YM}} I_{\mathrm{NLSM}}, \\
\mathrm{sGal} \sim I_{\mathrm{NLSM}} I_{\mathrm{NLSM}} .
\end{gathered}
$$

It is important to note that the integral (5) fully localizes on the delta functions, so in fact the amplitude is simply given by a sum over solutions to the scattering equations,

$$
A_{n}=\sum_{\sigma^{*}} J\left(\sigma^{*}\right) I_{L}\left(\sigma^{*}\right) I_{R}\left(\sigma^{*}\right),
$$

where $J$ is some Jacobian factor resulting from solving the delta functions. In practice, the scattering equations are nontrivial to solve (see [43-46] for developments), but they do not enter into our discussion.

\section{A. BCFW scaling}

We will use the following BCFW two particle shift $[16,18]$ :

$$
\begin{gathered}
e_{i} \rightarrow \hat{e}_{i}, \\
e_{j} \rightarrow \hat{e}_{j}+z p_{i} \frac{\hat{e}_{i} \cdot e_{j}}{p_{i} \cdot p_{j}}, \\
p_{i} \rightarrow p_{i}-z \hat{e}_{i}, \\
p_{j} \rightarrow p_{j}+z \hat{e}_{i},
\end{gathered}
$$

where

$$
\hat{e}_{i}=e_{i}-p_{i} \frac{e_{i} \cdot p_{j}}{p_{i} \cdot p_{j}}
$$

We also rescale in the world sheet coordinates,

$$
\begin{gathered}
\sigma_{i} \rightarrow(1+z) \sigma_{i}, \\
\sigma_{j} \rightarrow(1+z) \sigma_{j} .
\end{gathered}
$$

Under this shift, we find the scalings given in Table II.

The $\sigma$ shifts can be motivated by requiring the scattering equations corresponding to particles $i$ and $j$ to have an improved scaling $\mathcal{O}\left(z^{-1}\right)$ under an $[i, j\rangle$ shift [39],

$$
\begin{aligned}
\mathrm{SE}_{i} & =\sum_{k} \frac{p_{i} \cdot p_{k}}{\sigma_{i k}} \rightarrow \mathcal{O}\left(z^{-1}\right), \\
\mathrm{SE}_{j} & =\sum_{k} \frac{p_{j} \cdot p_{k}}{\sigma_{j k}} \rightarrow \mathcal{O}\left(z^{-1}\right) .
\end{aligned}
$$

Note that the other SEs do not have this behavior under the $[i, j\rangle$ shift. For instance, at four points under a $[1,2\rangle$ shift, the scattering equation for particle 3 becomes

$$
\begin{aligned}
\mathrm{SE}_{3}= & \sum_{k} \frac{p_{3} \cdot p_{k}}{\sigma_{3 k}} \rightarrow z^{0}\left(\frac{e_{1} \cdot p_{4}}{\sigma_{1}}-\frac{e_{1} \cdot p_{4}}{\sigma_{2}}+\frac{p_{1} \cdot p_{2}}{\sigma_{3}-\sigma_{4}}\right) \\
& +\mathcal{O}\left(z^{-1}\right)=\mathcal{O}\left(z^{0}\right) .
\end{aligned}
$$

While not mandatory, solving shifted scattering equations like Eq. (27) up to and including order $\mathcal{O}\left(z^{-1}\right)$ further improves the scaling of the YM integrand to $\mathcal{O}\left(z^{-4}\right)$.

It is interesting to note that the Laplace expansion of the reduced Pfaffian,

$$
\operatorname{Pf} M^{a b}=\sum(-1)^{j} m_{1 j} \operatorname{Pf} M^{a b 1 j},
$$

manifests the right BCFW scaling term by term in all shifts except those involving the "reduced" particles. Similarly, the cycle expansion of the full Pfaffian (which sums to zero) $[37,47,48]$ manifests both correct scaling and permutation invariance term by term,

$$
\operatorname{Pf} \Psi_{n}=\sum(-1)^{n-m} P_{i_{1} i_{2} \ldots i_{m}} .
$$


These special building blocks are given by

$$
P_{i_{1} i_{2} \ldots i_{r}}=\sum_{\left|I_{1}\right|=i_{i},\left|I_{2}\right|=i_{2}, \ldots\left|I_{r}\right|=i_{r}} \Psi_{I_{1}} \Psi_{I_{2}} \ldots \Psi_{I_{r}},
$$

where the $\Psi$ 's are gauge invariant, either via linearized field strengths or via the scattering equations,

$$
\begin{gathered}
\Psi_{(a)}=-\sum_{j \neq a} \frac{e_{a} \cdot p_{j}}{\sigma_{a j}}, \\
\Psi_{\left(a_{1} a_{2} \ldots a_{3}\right)}=\frac{\frac{1}{2} \operatorname{tr}\left(f_{a_{1}} f_{a_{2}} \ldots f_{a_{i}}\right)}{\sigma_{a_{1} a_{2}} \sigma_{a_{2} a_{3}} \ldots \sigma_{a_{i} a_{1}}}, \quad \text { with } \\
f_{a}^{\mu \nu}=p_{a}^{\mu} e_{a}^{\nu}-e_{a}^{\mu} p_{a}^{\nu} .
\end{gathered}
$$

Note that in this notation the integrand for $D F^{2}$ is precisely $P_{1111 \ldots 1}$.

\section{B. World sheet locality}

One property common to all integrands is what we will call world sheet locality: the correspondence between world sheet coordinates and spacetime dot products,

$$
\frac{x_{i} \cdot y_{i}}{\sigma_{i}-\sigma_{j}}
$$

with integrands given as a sum over products of such factors. This has an immediate effect on UV scaling: for pure scalar theories, no product of factors of the type (33) can scale worse than $\mathcal{O}\left(z^{0}\right)$, while for vector theories, because of multilinearity in polarization vectors, no term can scale worse than $\mathcal{O}\left(z^{-1}\right)$. Quite surprisingly, we will see that even this property follows directly from demanding improved scaling, as our initial Ansätze allow "nonlocal" factors,

$$
\frac{x_{i} \cdot y_{i}}{\sigma_{k}-\sigma_{l}}
$$

where $k$ and $l$ need not be related to $i$ and $j$.

\section{NONLINEAR SIGMA MODEL}

As mentioned before, we considering the object $\operatorname{Pf} A^{a b}$. Therefore, our Ansatz is defined by the following:

(i) Numerators are $(n / 2-1)$ dot products of $p_{i} \cdot p_{j}$.

(ii) Denominators are $(n / 2-1)$ factors of $\sigma_{i j}$.

For instance, the four point Ansatz is given by

$$
B_{4}=\sum_{i, j, k, l} a_{i j k l} \frac{p_{i} \cdot p_{j}}{\sigma_{k l}} .
$$

Then, after fixing some $a$ and $b$, we impose the following scalings:

$$
\begin{gathered}
{[i, j\rangle \sim \mathcal{O}\left(z^{-1}\right),} \\
{[i, a\rangle,[i, b\rangle,[a, b\rangle \sim \mathcal{O}\left(z^{0}\right)}
\end{gathered}
$$

for all $i, j \neq a, b$. In the four point case, choosing $a=3$ and $b=4$, only the $[1,2\rangle$ shift must scale as $\mathcal{O}\left(z^{-1}\right)$, and the other shifts must scale as $\mathcal{O}\left(z^{0}\right)$. By imposing this behavior, we find a unique solution,

$$
\operatorname{Pf} A_{4}^{34}=\frac{p_{1} \cdot p_{2}}{\sigma_{12}} .
$$

We can also perform the check at six points,

$$
B_{6}=\sum \frac{(p \cdot p)^{2}}{\left(\sigma_{i j}\right)^{2}} .
$$

This Ansatz already has around 5000 terms, but imposing the correct scaling, with $a=5$ and $b=6$, we find a unique solution,

$$
\operatorname{Pf} A_{6}^{56}=\frac{p_{1} \cdot p_{4} p_{2} \cdot p_{3}}{\sigma_{14} \sigma_{23}}-\frac{p_{1} \cdot p_{3} p_{2} \cdot p_{4}}{\sigma_{13} \sigma_{24}}+\frac{p_{1} \cdot p_{2} p_{3} \cdot p_{4}}{\sigma_{12} \sigma_{34}} .
$$

\section{YANG-MILLS}

We use the following Ansatz assumptions for $\operatorname{Pf} \Psi^{a b}$ :

(i) Numerators are multilinear in all polarization vectors, with mass dimension $[n-2]$.

(ii) Denominators are products of $(n-1) \sigma_{i j}$ factors.

(iii) Permutation invariance in $(n-2)$ particles.

At four points, such an Ansatz looks like

$$
B_{4}=a_{1} \frac{e_{1} \cdot e_{2} e_{3} \cdot p_{2} e_{4} \cdot p_{2}}{\sigma_{12} \sigma_{13} \sigma_{14}}+\ldots
$$

where again we note that the denominators are unrelated to the numerators. We then claim that the YM reduced Pfaffian of $\Psi^{a b}$ is uniquely fixed by the following scalings under BCFW shifts:

$$
\begin{gathered}
{[i, j\rangle,[i, a\rangle,[i, b\rangle \sim \mathcal{O}\left(z^{-2}\right),} \\
{[a, b\rangle,[b, a\rangle \sim \mathcal{O}\left(z^{-1}\right),}
\end{gathered}
$$

which is easily checked at four points.

At five points, the Ansatz is already too large to verify the claim, but there is a simple assumption which reduces the complexity: that each $\sigma_{i}$ should appear at least once per term. Then we are able to find a unique solution: the reduced five point Yang-Mills Pfaffian. 


\section{V. $D F^{2}$ THEORY}

We use the following assumptions:

(i) Numerators are multilinear in all polarization vectors, with mass dimension $[n]$; no $(e \cdot e)$ dot products allowed.

(ii) Denominators are products of $n \sigma_{i j}$ factors.

(iii) Permutation invariance in $n$ particles.

Therefore, an appropriate Ansatz has a form

$$
B_{4}=a_{1} \frac{e_{1} \cdot p_{2} e_{2} \cdot p_{1} e_{3} \cdot p_{2} e_{4} \cdot p_{2}}{\sigma_{12} \sigma_{13} \sigma_{14} \sigma_{34}}+\ldots
$$

Then we claim that the $D F^{2}$ integrand is uniquely fixed by a BCFW scaling of $\mathcal{O}\left(z^{-2}\right)$ under all two particle shifts. This is easily verified at four points, but at five points we again need to impose the presence of every $\sigma_{i}$ in each term, which allows us to check a somewhat weaker claim.

It is interesting to note that if we also allow $(e \cdot e)$ factors in the Ansatz, we obtain new solutions which include all the $P_{i_{1} i_{2} \ldots i_{r}}$ building blocks of the cycle expansion of the Pfaffian in Eq. (30). At four points, we only obtain one extra solution, which can be eliminated either by imposing gauge invariance (on the support of the scattering equations), or by demanding $\mathcal{O}\left(z^{1}\right)$ scaling under the transformation

$$
\begin{aligned}
& p_{i} \rightarrow z p_{i}, \\
& \sigma_{i} \rightarrow z \sigma_{i} .
\end{aligned}
$$

It remains an open question whether this is still true at higher points and whether there exist any constraints which select the linear combinations of the $P_{i_{1} i_{2} \ldots i_{r}}$ that correspond to $F^{3}$-type interactions [37].

\section{A. Spacetime $D F^{2}$}

In spacetime, we are back to usual propagators, which in the case of $D F^{2}$ [42], a nonunitary theory, come in two types: gluon propagators, which are sum of consecutive momenta $\left(\sum p_{i}\right)^{4}$, and scalar propagators, which can be sums of nonconsecutive momenta $\left(\sum p_{i}\right)^{2}$, since the scalar color structure allows nonplanar interactions. Like the CHY integrand, we do not allow dot products $(e \cdot e)$, but only $(e \cdot p)$ and $(p \cdot p)$. A four point Ansatz is given by

$$
A_{4}=\frac{n_{s}\left(p^{6}\right)}{s^{2}}+\frac{n_{t}\left(p^{6}\right)}{t^{2}}+\frac{n_{u}\left(p^{4}\right)}{u},
$$

where $n\left(p^{k}\right)$ are numerators of mass dimension $k$. Imposing gauge invariance in all four particles,

$$
e_{i} \rightarrow p_{i} \Rightarrow A_{4}=0
$$

we find a unique result, the $D F^{2}$ amplitude. This is similar to the YM uniqueness from gauge invariance $[14,15]$. Note that the numerators cannot be independently gauge invariant in all particles, because they are only functions of $e \cdot p$ and $p \cdot p$. A polynomial of the form $f_{n}\left((e \cdot p)^{n},(p \cdot p)^{k}\right)$ may only be gauge invariant in $k$ particles, while $D F^{2}$ numerators are of the form $f_{n}\left((e \cdot p)^{n},(p \cdot p)^{n-3}\right)$. Therefore, only a sum of diagrams can be fully gauge invariant, again similar to the case of Yang-Mills.

Finally, we also find that the BCFW scalings,

$$
\mathcal{O}\left(z^{1}\right) \text { for adjacent shifts, }
$$

$$
\mathcal{O}\left(z^{0}\right) \text { for non-adjacent shifts, }
$$

uniquely fix the $D F^{2}$ amplitudes, at least up to $n=5$.

\section{CONCLUSION}

We have shown that the constraining power of BCFW shifts is sufficient to fully determine the main ingredients of the CHY formalism, further demonstrating the surprising universality of enhanced UV scaling. While the claim for $\mathrm{YM}$ and $D F^{2}$ theory only holds if also assuming permutation invariance, it appears that this assumption can be replaced with another type of UV scaling: single particle shifts $[i\rangle$, introduced in [16]. These shifts are simply given by

$$
\begin{gathered}
e_{i} \rightarrow \hat{e}_{i}, \\
p_{i} \rightarrow p_{1}-z \hat{e}_{i}, \\
\sigma_{i} \rightarrow z \sigma_{i} .
\end{gathered}
$$

At four points, we find that the usual two particle shifts, together with the single particle scalings,

$$
\begin{gathered}
\mathrm{YM}:[i\rangle \sim \mathcal{O}\left(z^{n-5}\right) \text { and }[a\rangle,[b\rangle \sim \mathcal{O}\left(z^{n-4}\right), \\
D F^{2}:[i\rangle \sim \mathcal{O}\left(z^{n-3}\right)
\end{gathered}
$$

are enough to fully constrain the respective integrands, allowing a definition purely in terms of UV scalings with no assumption of permutation invariance.

Meanwhile, the $\mathcal{O}\left(z^{-2}\right)$ scaling also guarantees the lack of a pole at infinity, and so the integrands may be rebuilt from finite residues. ${ }^{1}$ It is worth mentioning that the $\mathcal{O}\left(z^{-2}\right)$

\footnotetext{
${ }^{1}$ The $\mathrm{BCFW}$ reconstruction of $\mathrm{CHY}$ was investigated in the original proof of this formalism in Ref. [39], but factorization based on the existence of scattering equations was crucial in that approach.
} 
of the integrands also implies the existence of so-called bonus relations [23] between residues.

A related theory is the so-called " $D F^{2}+\mathrm{YM}$," relevant to the bosonic string, and which also has a $\mathrm{CHY}$ construction [49-51]. It would be interesting to investigate whether there exist (purely field theoretic) consistency conditions which determine this object as well.

\section{ACKNOWLEDGMENTS}

The author would like to thank John Joseph Carrasco, Song He, and Yong Zhang for discussions, and is grateful to the Institute of Theoretical Physics, Chinese Academy of Sciences, Beijing, where this work was initiated. The author was supported by the European Research Council under ERC-STG-639729, Strategic Predictions for Quantum Field Theories.
[1] R. Britto, F. Cachazo, B. Feng, and E. Witten, Direct Proof of Tree-Level Recursion Relation in Yang-Mills Theory, Phys. Rev. Lett. 94, 181602 (2005).

[2] Z. Bern, L. J. Dixon, D. C. Dunbar, and D. A. Kosower, One loop $n$-point gauge theory amplitudes, unitarity and collinear limits, Nucl. Phys. B425, 217 (1994).

[3] N. Arkani-Hamed and J. Trnka, The amplituhedron, J. High Energy Phys. 10 (2014) 030.

[4] N. Arkani-Hamed, Y. Bai, S. He, and G. Yan, Scattering forms and the positive geometry of kinematics, color and the worldsheet, J. High Energy Phys. 05 (2018) 096.

[5] Z. Bern, J. J. M. Carrasco, and H. Johansson, New relations for gauge-theory amplitudes, Phys. Rev. D 78, 085011 (2008).

[6] Z. Bern, J. J. M. Carrasco, and H. Johansson, Perturbative Quantum Gravity as a Double Copy of Gauge Theory, Phys. Rev. Lett. 105, 061602 (2010).

[7] Z. Bern, J. J. Carrasco, M. Chiodaroli, H. Johansson, and R. Roiban, The duality between color and kinematics and its applications, arXiv:1909.01358.

[8] J. J. M. Carrasco, L. Rodina, Z. Yin, and S. Zekioglu, Simple encoding of higher derivative gauge and gravity counterterms, arXiv:1910.12850.

[9] C. Cheung, C.-H. Shen, and C. Wen, Unifying relations for scattering amplitudes, J. High Energy Phys. 02 (2018) 095.

[10] C. Cheung and G. N. Remmen, Entanglement and the double copy, J. High Energy Phys. 05 (2020) 100.

[11] S. L. Adler, Consistency conditions on the strong interactions implied by a partially conserved axial vector current, Phys. Rev. 137, B1022 (1965).

[12] C. Cheung, K. Kampf, J. Novotny, and J. Trnka, Effective Field Theories from Soft Limits of Scattering Amplitudes, Phys. Rev. Lett. 114, 221602 (2015).

[13] C. Cheung, K. Kampf, J. Novotny, C.-H. Shen, and J. Trnka, A periodic table of effective field theories, J. High Energy Phys. 02 (2017) 020.

[14] N. Arkani-Hamed, L. Rodina, and J. Trnka, Locality and Unitarity of Scattering Amplitudes from Singularities and Gauge Invariance, Phys. Rev. Lett. 120, 231602 (2018).

[15] L. Rodina, Uniqueness from gauge invariance and the Adler zero, J. High Energy Phys. 09 (2019) 084.

[16] J. J. M. Carrasco and L. Rodina, UV considerations on scattering amplitudes in a web of theories, Phys. Rev. D 100, 125007 (2019).
[17] L. Rodina, Scattering Amplitudes from Soft Theorems and Infrared Behavior, Phys. Rev. Lett. 122, 071601 (2019).

[18] L. Rodina, Uniqueness from locality and BCFW shifts, J. High Energy Phys. 09 (2019) 078.

[19] N. Arkani-Hamed and J. Kaplan, On tree amplitudes in gauge theory and gravity, J. High Energy Phys. 04 (2008) 076.

[20] N. Arkani-Hamed, F. Cachazo, and J. Kaplan, What is the simplest quantum field theory? J. High Energy Phys. 09 (2010) 016.

[21] P. C. Schuster and N. Toro, Constructing the tree-level Yang-Mills S-matrix using complex factorization, J. High Energy Phys. 06 (2009) 079.

[22] D. A. McGady and L. Rodina, Recursion relations for graviton scattering amplitudes from Bose symmetry and bonus scaling laws, Phys. Rev. D 91, 105010 (2015).

[23] M. Spradlin, A. Volovich, and C. Wen, Three applications of a bonus relation for gravity amplitudes, Phys. Lett. B 674, 69 (2009).

[24] K. Kampf, J. Novotny, and J. Trnka, Tree-level amplitudes in the nonlinear sigma model, J. High Energy Phys. 05 (2013) 032.

[25] K. Kampf, J. Novotny, and J. Trnka, Recursion relations for tree-level amplitudes in the $S U(N)$ nonlinear sigma model, Phys. Rev. D 87, 081701 (2013).

[26] C. Cheung, C.-H. Shen, and J. Trnka, Simple recursion relations for general field theories, J. High Energy Phys. 06 (2015) 118.

[27] H. Luo and C. Wen, Recursion relations from soft theorems, J. High Energy Phys. 03 (2016) 088.

[28] F. Cachazo, P. Cha, and S. Mizera, Extensions of theories from soft limits, J. High Energy Phys. 06 (2016) 170.

[29] I. Low and Z. Yin, Soft bootstrap and effective field theories, J. High Energy Phys. 11 (2019) 078.

[30] H. Elvang, M. Hadjiantonis, C. R. T. Jones, and S. Paranjape, Soft bootstrap and supersymmetry, J. High Energy Phys. 01 (2019) 195.

[31] A. Edison, E. Herrmann, J. Parra-Martinez, and J. Trnka, Gravity loop integrands from the ultraviolet, arXiv:1909 .02003 .

[32] F. Cachazo, S. He, and E. Y. Yuan, Scattering equations and Kawai-Lewellen-Tye orthogonality, Phys. Rev. D 90, 065001 (2014). 
[33] F. Cachazo, S. He, and E. Y. Yuan, Scattering of Massless Particles in Arbitrary Dimensions, Phys. Rev. Lett. 113, 171601 (2014).

[34] F. Cachazo, S. He, and E. Y. Yuan, Scattering of massless particles: Scalars, gluons and gravitons, J. High Energy Phys. 07 (2014) 033.

[35] F. Cachazo, S. He, and E. Y. Yuan, Einstein-Yang-Mills scattering amplitudes from scattering equations, J. High Energy Phys. 01 (2015) 121.

[36] F. Cachazo, S. He, and E. Y. Yuan, Scattering equations and matrices: From Einstein to Yang-Mills, DBI and NLSM, J. High Energy Phys. 07 (2015) 149.

[37] S. He and Y. Zhang, New formulas for amplitudes from higher-dimensional operators, J. High Energy Phys. 02 (2017) 019.

[38] T. Azevedo and O. T. Engelund, Ambitwistor formulations of $\mathrm{R}^{2}$ gravity and $(\mathrm{DF})^{2}$ gauge theories, J. High Energy Phys. 11 (2017) 052.

[39] L. Dolan and P. Goddard, Proof of the formula of Cachazo, He and Yuan for Yang-Mills tree amplitudes in arbitrary dimension, J. High Energy Phys. 05 (2014) 010.

[40] L. Mason and D. Skinner, Ambitwistor strings and the scattering equations, J. High Energy Phys. 07 (2014) 048.

[41] E. Casali, Y. Geyer, L. Mason, R. Monteiro, and K. A. Roehrig, New Ambitwistor string theories, J. High Energy Phys. 11 (2015) 038.
[42] H. Johansson and J. Nohle, Conformal gravity from gauge theory, arXiv:1707.02965.

[43] C. Baadsgaard, N. E. J. Bjerrum-Bohr, J. L. Bourjaily, and P. H. Damgaard, Integration rules for scattering equations, J. High Energy Phys. 09 (2015) 129.

[44] N. E. J. Bjerrum-Bohr, J. L. Bourjaily, P. H. Damgaard, and B. Feng, Analytic representations of Yang-Mills amplitudes, Nucl. Phys. B913, 964 (2016).

[45] K. Zhou, J. Rao, and B. Feng, Derivation of Feynman rules for higher order poles using cross-ratio identities in CHY construction, J. High Energy Phys. 06 (2017) 091.

[46] C. S. Lam, Pfaffian diagrams for gluon tree amplitudes, Phys. Rev. D 98, 076002 (2018).

[47] C. S. Lam and Y.-P. Yao, Evaluation of the Cachazo-HeYuan gauge amplitude, Phys. Rev. D 93, 105008 (2016).

[48] R. Huang, Y.-J. Du, and B. Feng, Understanding the cancelation of double poles in the Pfaffian of CHYformulism, J. High Energy Phys. 06 (2017) 133.

[49] S. He, F. Teng, and Y. Zhang, String Amplitudes from FieldTheory Amplitudes and Vice Versa, Phys. Rev. Lett. 122, 211603 (2019).

[50] T. Azevedo, M. Chiodaroli, H. Johansson, and O. Schlotterer, Heterotic and bosonic string amplitudes via field theory, J. High Energy Phys. 10 (2018) 012.

[51] S. He, F. Teng, and Y. Zhang, String correlators: Recursive expansion, integration-by-parts and scattering equations, J. High Energy Phys. 09 (2019) 085. 